\title{
Automatic detection of mode mixing in empirical mode decomposition using non-stationarity detection: application to selecting IMFs of interest and denoising
}

\author{
Jeremy Terrien ${ }^{1 *}$, Catherine Marque ${ }^{2}$ and Brynjar Karlsson ${ }^{3}$
}

\begin{abstract}
Empirical mode decomposition splits a signal into several intrinsic mode functions (IMF). An algorithm for the automatic selection of the modes containing the signal of interest was recently proposed. This algorithm is based on statistical analysis describing the noise repartition between IMFs. This algorithm uses an estimate of the signal noise content from the energy of the first IMF, which is supposed to contain a specific part of the total noise and to contain noise only. Mode mixing can give rise to an over-estimation of the noise in the signal. This can lead to more IMFs to be considered as containing only noise and to be erroneously discarded before reconstruction. We propose to use mode mixing detection based on a stationarity test applied to the first IMF. In case of mode mixing, we propose to correct the noise estimation by extracting from the first IMF the part corresponding to the signal of interest. The results obtained with synthetic signals as well as with real mechanical and biomedical signals demonstrate a good performance of the approach proposed here. The first two modes do lose some of their IMF properties in the process. We offer some comments on how these properties can be recovered if needed.
\end{abstract}

Keywords: empirical mode decomposition, mode mixing, non-stationary signal detection, mode selection, denoising

\section{Introduction}

The use of the Hilbert-Huang transform is becoming increasingly popular in various domains of research. The method is based on the empirical mode decomposition (EMD), which allows the iterative decomposition of a signal into a series of functions that are referred to as intrinsic mode functions (IMF) [1].

Noise in the signal of interest will result in the contamination of each mode by a part of the noise. The study of the spectral content and of the statistical characteristics of the EMD modes, computed from Gaussian white noise, led to the definition of a model that can quantify the information content of each IMF [2]. This model was generalized by Flandrin et al. [3] in the case of fractional Gaussian noise. These models can be used

\footnotetext{
* Correspondence: jterrien@utc.fr

'Department of electronics, Université de Technologie de Compiègne,

Compiègne Cedex, F60205, France

Full list of author information is available at the end of the article
}

for denoising or for the suppression of an unwanted baseline wander (detrending) of the signal of interest. A fundamental hypothesis that is made, when these models are used for the above application, is that the first IMF contains a specific part of the noise as derived from the above models. An estimate of the energy of the first IMF is then used to adapt the denoising algorithm to the signal and to its corrupting noise. If an error is made in estimating the actual level of noise from the first IMF, it can seriously decrease the performance of the algorithm.

One source of such error can be mode mixing, which corresponds to the alternating presence of several components of the signal of interest on the same IMF [4]. In our application, this mixing mainly involves the high frequency part of the noise and the first high frequency component of the signal. This type of mixing will invalidate the fundamental assumption of the method that

\section{SpringerOpen ${ }^{\circ}$}

(C) 2011 Terrien et al; licensee Springer. This is an Open Access article distributed under the terms of the Creative Commons Attribution License (http://creativecommons.org/licenses/by/2.0), which permits unrestricted use, distribution, and reproduction in any medium, provided the original work is properly cited. 
the first IMF contains only noise and lead to an overestimation of the level of noise in the signal.

An algorithm that can prevent mode mixing has recently been proposed [4]. This algorithm is, however, limited to the EMD analysis of signals composed of pure sinusoids. When used on signals that contain nonstationary elements, like those commonly found in real signals (especially those of biomedical or mechanical origin), this algorithm does not perform well. Several authors have, therefore, developed algorithms that are specifically adapted to their signals. An example of this is the algorithm proposed by Blanco-Velasco et al. [5] for the analysis of ECG. The algorithm presented here does not make any a priori hypothesis concerning the nature of the signal components and therefore can be considered as a very general alternative to existing algorithms used for this purpose.

We first present shortly the classic algorithm for selecting the pertinent modes of a signal. Then, we introduce an improvement of this algorithm, using a stationarity test on the first IMF that allows us to make a more robust estimate of the statistical cutoff values used for selecting IMFs. We then present an evaluation of the performance of our algorithm when applied to synthetic signals and real signals obtained from mechanical and biomedical systems.

\section{Algorithms for IMF selection}

We consider that the EMD of a signal $x[n](n=1, \ldots, N)$ results in a set of $K$ IMFs $d_{k}[n](k=1, \ldots, K)$ and a residual signal $r[n]$. The signal $x$ is considered to be composed of a signal $s[n]$ that is corrupted with a fractional Gaussian noise $G n f_{H}[n]$. The signal $G n f_{H}[n]$ is stationary by definition.

\section{Classic approach}

A statistical model of the energy distribution of noise between IMFs has been derived by studying the energy in the modes of a fractional Gaussian noise after EMD [2]. This model makes it possible to define a statistical cutoff value, below which the IMF is not considered as a preponderant part of the signal. With this model, the first IMF of a signal contaminated with noise is always considered to be purely noise. The energy of the first IMF is simply:

$$
W_{H}[1]=\sum_{n=1}^{N} d_{1}^{2}[n]
$$

The energy of the noise in the other IMFs is then deduced, for a given Hurst exponent $H$, as:

$$
W_{H}[k]=C_{H} \rho_{H}^{-2(1-H) k}, \quad K \geq 2
$$

with $C_{H}=W_{H}[1] / \beta H, \beta_{H}=0.719$ for $H=0.5$, and $\rho_{H}$ $=2.01+0.2 \times(H-0.5)+0.12 \times(H-0.5)^{2}$.

Additionally, we can define with this model a confidence interval $T_{H}[k]$. The decomposition of fractional Gaussian noise yields an energy distribution for a given IMF over several noise realizations. The confidence interval corresponds to an energy limit inside which $x \%$ (e.g. 95 or $99 \%$ ) of the noise realizations are below this value. The confidence interval plays the role of a statistical threshold. There is a linear relationship between the logarithm of the confidence interval and the number of the IMF, $k$, given by (3):

$$
\log _{2}\left(\log _{2}\left(T_{H}[k]\right) / W_{H}[k]\right)=a_{H} k+b_{H}
$$

where, in the case of $H=0.5$ and for a confidence interval of 99\%: $a_{H}=0.45$ and $b_{H}=-1.95$. Values of $\beta_{H}$, $a_{H}$, and $b_{H}$ can be found in [3] for other values of $H$.

The selection algorithm proposed by Flandrin et al. is the following:

1. Estimate the energy of the noise $W_{H}[1]$ on $d_{1}[n]$

2. Estimate the pure noise content, $W_{H}[2, \ldots, k]$, using the estimate in (1)

3. Estimate the confidence interval $T_{H}[1, \ldots, k]$

4. Compare the energy of the IMFs 2 to $K$ with the confidence interval

5. All the IMFs that have energy greater than the confidence interval are considered to be components of the signal and not to be pure noise.

If the desired result is to remove the noise, the signal is partially reconstructed by summing the selected IMFs and the residual $r[n]$.

\section{The proposed approach}

In the absence of any mode mixing, the first IMF $\left(d_{1}[n]\right)$ contains noise only and is therefore stationary. If some mode mixing is present, $d_{1}[n]$ is not stationary. We propose here to detect mode mixing using a test of stationarity. To test the stationarity of the first IMF, we used an algorithm developed by Xiao et al. [6]. It is a general and robust method that only requires the user to choose the level of significance for the detection. The value we used in this work is $p=0.05$.

If mode mixing is present in the first IMF $\left(d_{1}[n]\right)$, it becomes necessary to distinguish between the part of its energy due to noise and the part due to the signal mixed in with the IMF. To do this, we simply consider here the first IMF to be a noisy signal. We therefore apply a wavelet filter, $f_{\text {wavelet, }}$ to separate the part due to the original signal, $s[n]$, from the part due to noise, $b[n]$.

The modified algorithm is thus the following: 
1. Test if $d_{1}[n]$ is stationary, if yes go to (4)

2. Extract from $d_{1}[n]$ the part that is noise $b[n]$ and the part that is signal $s[n]$ by wavelet filtering $\left(f_{\text {wavelet }}\right.$

$(x))$

$$
\begin{aligned}
& \text { a. } s[n]=f_{\text {wavelet }}\left(d_{1}[n]\right) \\
& \text { b. } b[n]=d_{1}[n]-s[n]
\end{aligned}
$$

3. Mode de-mixing

$$
\begin{aligned}
& \text { a. } d_{1}[n]=b[n] \\
& \text { b. } d_{2}[n]=d_{2}[n]+s[n]
\end{aligned}
$$

4. Apply the classic algorithm for selecting IMFs as described in the previous section.

Every kind of wavelet filter could be used, e.g. discrete wavelet filter or wavelet packet filter. The choice depends on the characteristics of the signal of interest. In this work we chose to use a discrete wavelet filter, which is the simplest of all possible wavelet filters.

\section{Evaluation of the new algorithm 1. Signals}

In this work, we tested our algorithm on several kinds of signals: a purely synthetic signal made from three Gabor atoms, a signal generated by a vibrating ball bearing, and finally, an ECG signal generated using a model proposed by McSharry et al. [7]. We evaluated the robustness of our method by adding Gaussian white noise to these signals to obtain SNR values of 20,18, 16,14 , and $12 \mathrm{~dB}$. For each SNR, we studied 500 realizations.

\section{IMF selection or denoising performances}

We used, as an evaluation criterion, the normalized percent mean square error (NPMSE) between the original signal without noise and the reconstructed signal. NPMSE is defined as:

$$
\mathrm{NPMSE}=100 \times \frac{\sum_{i=1}^{n}(x[i]-\hat{x}[i])^{2}}{\sum_{i=1}^{n} x[i]^{2}}
$$

where $x$ and $\hat{x}$ are the original and the denoised signal, respectively.

We compared the 'classic' method (from [2]) to the improved approach proposed in this paper. We also compared the classic method to our approach, but without adding to the reconstruction the part of the first IMF corresponding to the signal (step 3b). In that way, we tested separately the effect of the cutoff values correction, on the reconstruction of the signal.

We also studied the influence of the wavelet choice and of the number of decomposition levels (step 2a) on the performance of the new algorithm proposed. We tested the Daubechie of order 8, Meyer and Haar wavelets and 5, 8, and 10 decomposition levels. In all experiments, the noise estimation in the decomposition levels was done using the heuristic variant of the Stein's unbiased rule. A soft threshold was then applied on each decomposition level before the reconstruction of the denoised original signal.

\section{IMF characteristics}

The IMFs and the residual signal from the first IMF are summed up after the application of the algorithm to reconstruct the signal. In the de-mixing step of the proposed algorithm, we chose to add the part of the signal extracted from the first IMF to the second IMF. This addition of a part of the first IMF to the second can make the second IMF lose some of its characteristics as a true IMF. Although this does not affect the process of signal reconstruction and does not diminish the quality of the reconstructed signal, it may be important to preserve the characteristics of the EMD decomposition, if further processing of the signal is to be done. As an example we may be interested in the Hilbert-Huang spectrum of the different IMFs.

To clarify the issue of how much our algorithms have induced the second IMF to differ from a true IMF, we created a method to quantify how close the modified IMF is to a true IMF.

If a signal $(s(t))$ has all the properties of an IMF, its EMD will result in one and only one IMF that will be identical to the signal itself. In this case, the first IMF's energy relative to the signal will be $100 \%$. If the analyzed signal is not a pure IMF, its decomposition is going to result in several IMFs. Therefore, the energy of the first IMF, obtained from the signal, will be increasingly smaller as the signal is further away from being a pure IMF.

We therefore defined an 'IMF-likeness' measure to quantify the modification to the second IMF. We first applied EMD to the $\mathrm{IMF}_{\mathrm{i}}$ (modified second IMF) to be tested. Then, we computed the energy of the first IMF, obtained from this decomposition of $\mathrm{IMF}_{\mathrm{i}}$, divided by its original energy (energy of $\mathrm{IMF}_{\mathrm{i}}$ ). If $\mathrm{IMF}_{\mathrm{i}}$ still has the characteristics of an IMF, this ratio gives a number close to 1 , but gives a smaller value when $\mathrm{IMF}_{\mathrm{i}}$ is less like an IMF.

\section{Results}

Figure 1 shows an example of the EMD obtained on a synthetic signal composed of three Gabor atoms with 12 $\mathrm{dB}$ noise added. We can clearly see that the first IMF contains mode mixing. Indeed, a part of the signal is clearly evident in this mode. The energy of the IMF that corresponds to the signal is stronger than the noise energy. It will, therefore, lead to an over-estimation of the noise contained in the original signal.

Figure 2 presents the estimation of the noise model and the associated confidence interval $\left(T_{\mathrm{H}}\right)$ by means of the classic algorithm (upper panel), based on the assumption that the first IMF contains noise only, and by using our method (lower panel). We can easily notice 


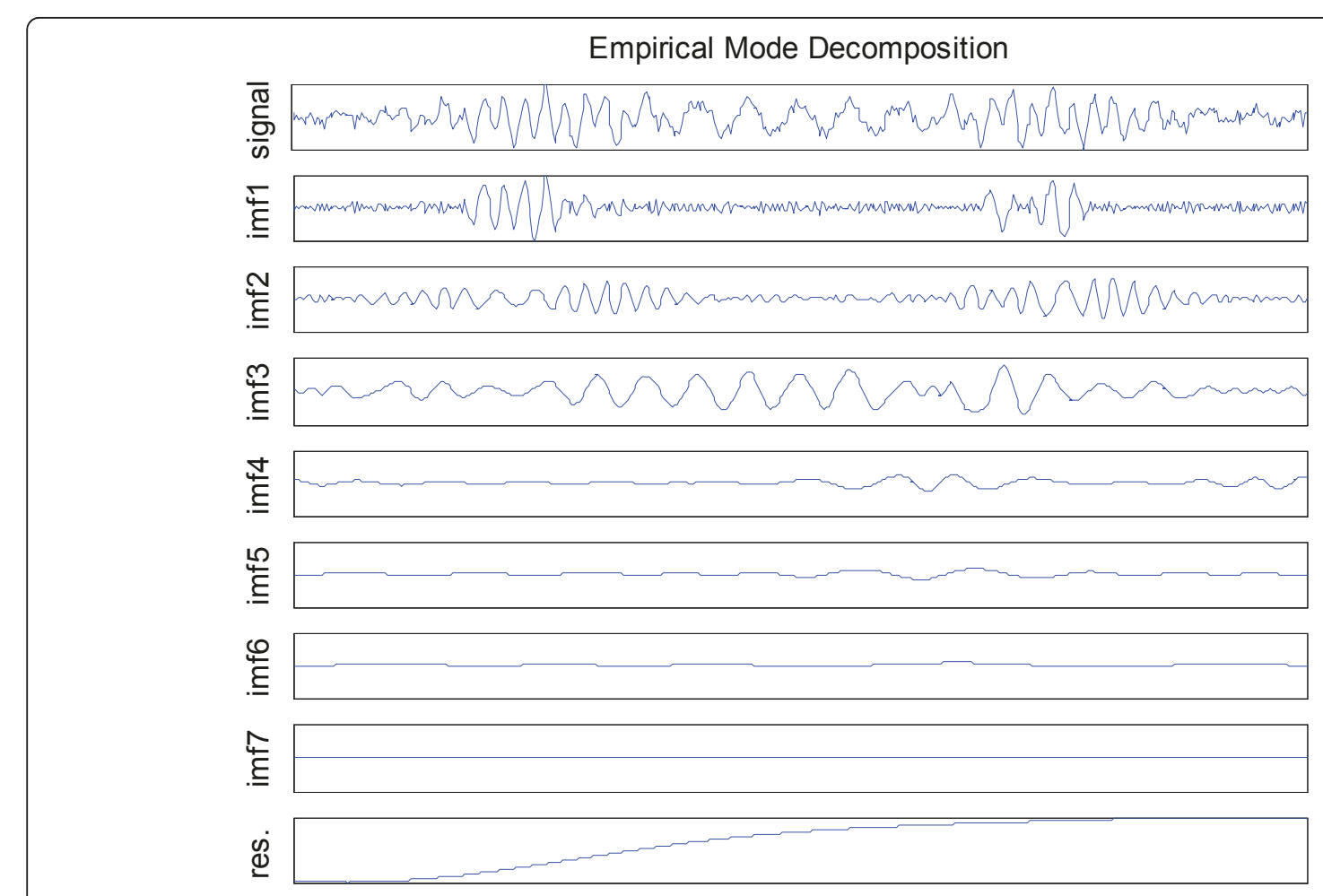

Figure $1 \mathrm{EMD}$ of a synthetic signal with $12 \mathrm{~dB}$ SNR.
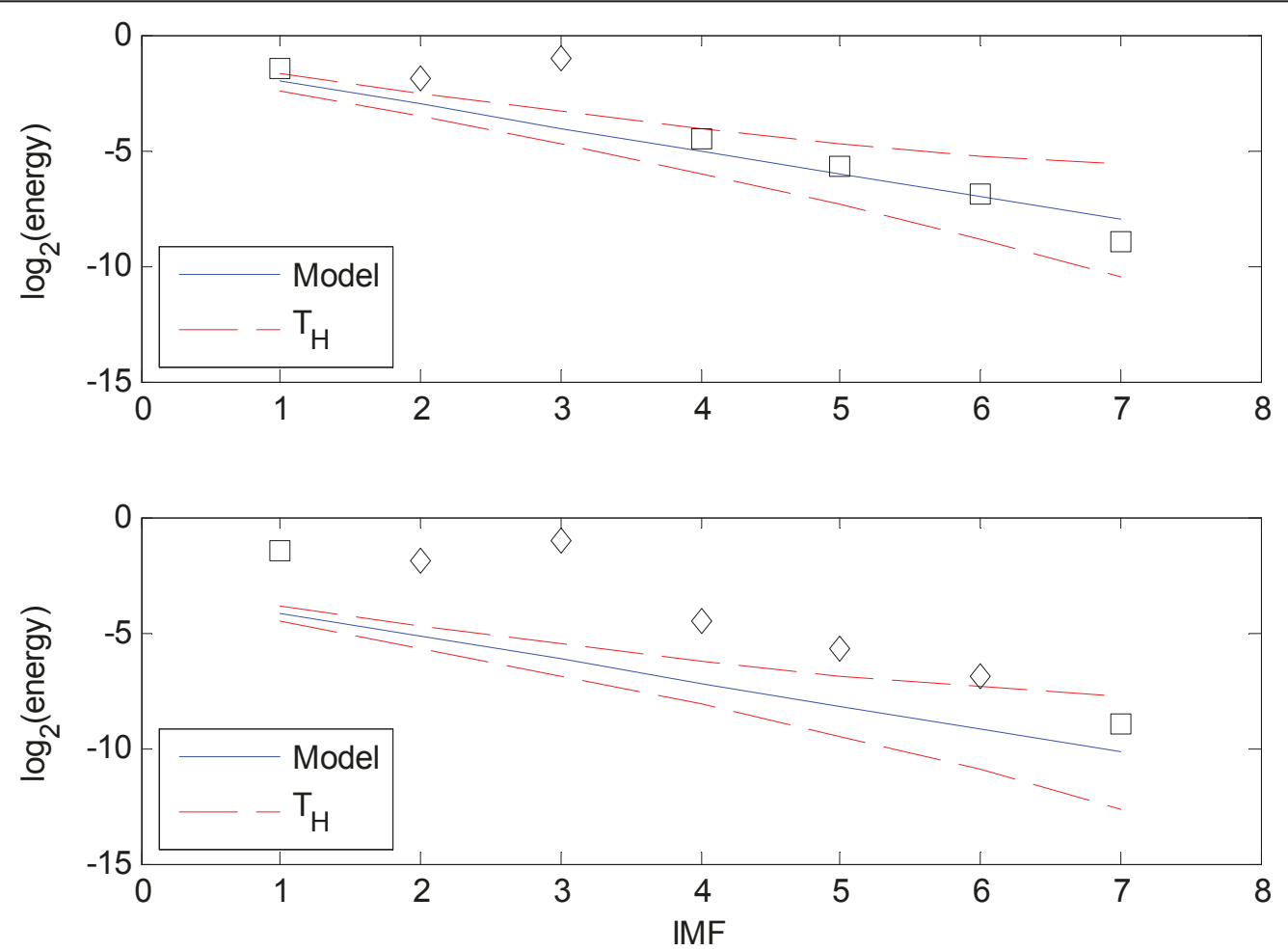

Figure 2 An example of the selection of modes of the signal presented in Figure 1 by the classic algorithm (above) and by the method presented in this work (below). The estimated model and the associated confidence interval $\left(T_{H}\right)$ are presented as a straight line and a dashed line, respectively. The selected IMFs are diamond shaped and the ones that are rejected are represented by squares. 
that the over-estimation of the noise level leads to the rejection of IMFs 4 to 6 , even though they clearly contain a part of the original signal. To correct this, if we detect the presence of mode mixing in the first IMF, we propose, in the new algorithm, to separate the part corresponding to the signal from that corresponding to noise. Figure 3 presents the mode de-mixing result obtained by our algorithm. The correction of the noise level estimation results in the appropriate selection of IMFs 4 to 6 (Figure 2, lower panel).

Figure 4 presents the results of the robustness analysis for the three algorithms tested: the classical method (1), the de-mixing of IMF1 only (2), the de-mixing plus addition of the IMF1 signal to IMF2 (3). Correcting the noise level estimation in IMF1 (method 2) induces a reduction of the reconstruction error when compared to method 1 (Figure 4, upper panel). In addition, if the signal contained in the IMF1 is taken into account in IMF2, the level of reconstruction error drops to very small values, for all values of SNR. In all cases, the results of our method are better than those obtained with the classical method. We also note that the standard deviation is smaller for our methods in all cases.

Figure 4 (lower panel) presents the influence of the choice of wavelet. We can notice that the different wavelets influence the quality of the results. In particular, Haar's wavelet provides the worst results. Meyer's and Daubechie's wavelets give similar results even if the error is slightly lower for Meyer's wavelet. The error obtained using either of these two wavelets is lower than $5 \%$. The number of levels of the wavelet decomposition had a negligible effect on the quality of the reconstruction (results not shown). This can be explained by the relatively great difference in the frequency content of the signal and of the noise contained in the first IMF of the synthetic signal under study.

When applied to the ball bearing signal (Figure 5) or to the simulated ECG signals (Figure 6), the results of the algorithms are similar to those obtained for the synthetic signal. The standard deviations are, however, greater for the ECG signals than those obtained on the other signals. This is most probably due to the high variability of the number of QRS complexes contained in the first IMF in different realizations of the noisy signal. For these signals, the number of levels of the wavelet decomposition also has a very small effect on the reconstruction error, even though the effect is higher than the one observed for synthetic signals.

Table 1 presents the results obtained for the 'IMF likeness measure' for the different signals used in this study, with a $16 \mathrm{~dB}$ SNR. We can generally notice that the signal obtained by removing the noise from the first IMF (first denoised IMF) is not an IMF according to our measure (Table 1, column A). It is, however, fairly

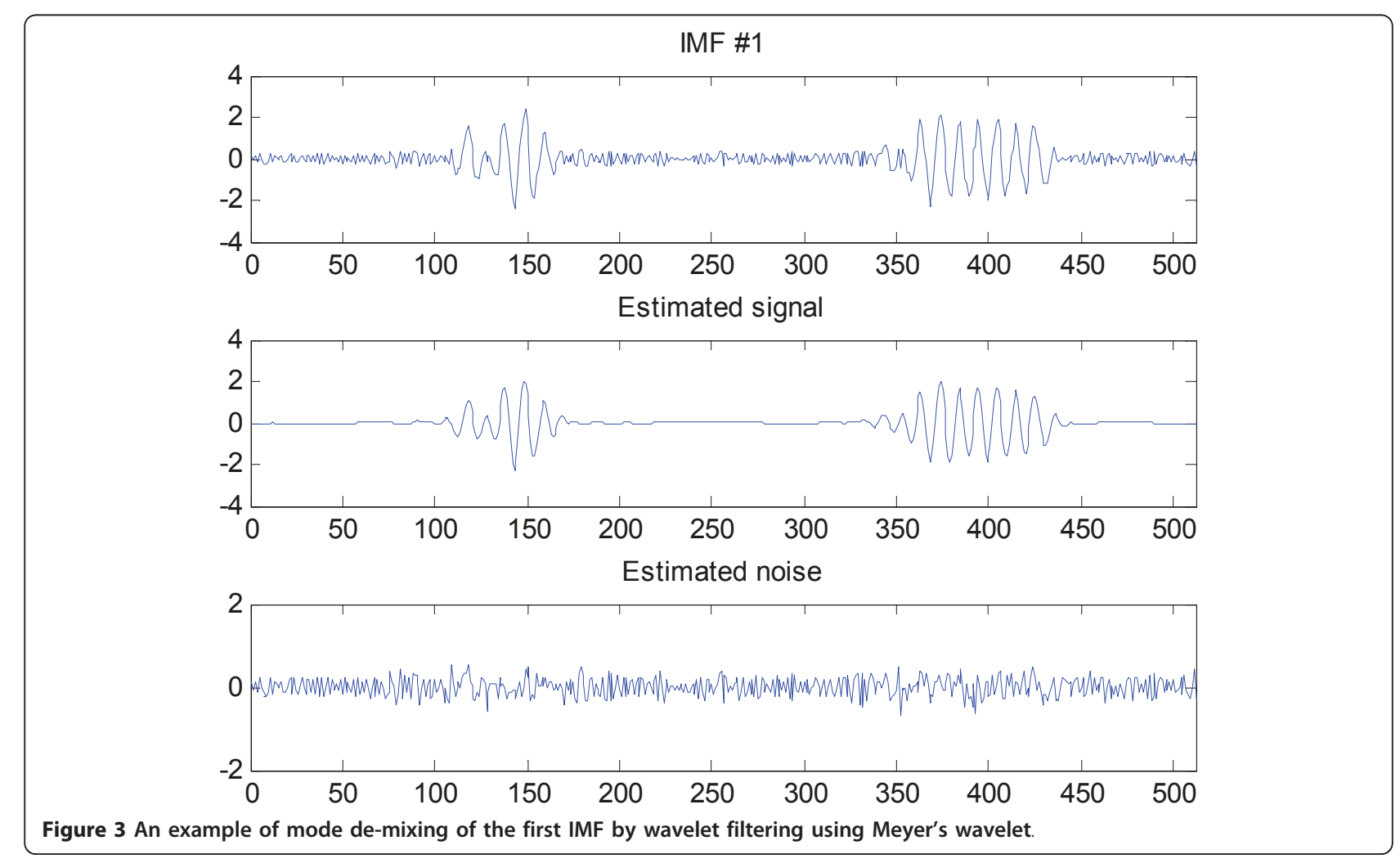



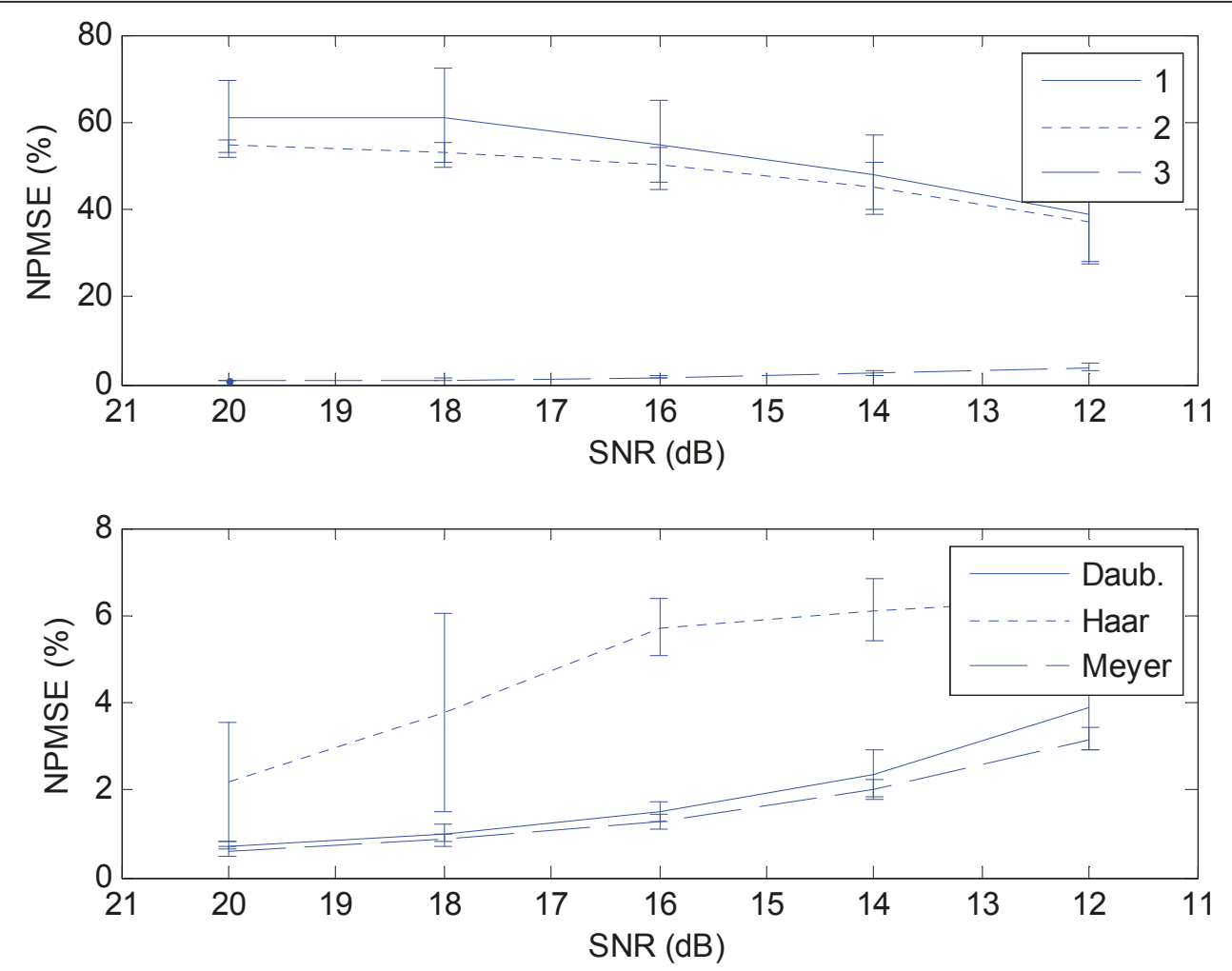

Figure 4 Results on the synthetic signal composed of three Gabor atoms plus noise. Above: The evolution of the percentage of mean square error (NPMSE) $( \pm \sigma)$ as obtained by using the classic method (1), by only correcting the estimate of noise in the first IMF (2) and by correcting the estimate of noise in the first IMF and plus modification of the second IMF (3). Below: Comparison of the NPMSE obtained using the wavelets Daubechie (Daub.), Haar and Meyer.

close to being an IMF except for the case of the ECG signals. For the mechanical (ball bearing) and ECG signals, adding the signal part of the first IMF (first denoised IMF) to the second IMF gives better IMF-like characteristics, according to our measure (Table 1, column B). Only for the synthetic signals, this addition reduces the IMF likeness measure.

\section{Conclusion}

We have proposed in this paper an improvement of an existing algorithm used for selecting the IMFs of interest, which is useful in case of mode mixing that can affect the performances of the original algorithm. The existing algorithm uses the first IMF to provide an estimate of the noise contained in each of the IMFs of an EMD. The presence of a mixing of modes introduces errors to this estimation and may lead to a bad selection of the IMFs of interest. Our approach is based on the detection of mode mixing by a test of stationarity on the first IMF, and on the extraction of the part of the first IMF that corresponds to pure noise. The choice of the stationarity test and of the noise extraction algorithm has to be made according to the specific signal of interest. In this work, we chose a stationarity test based on the statistical study of time-frequency surrogates of the signal, and a wavelet filter for separating noise from signal in the first IMF. These choices present the advantage of not making a priori assumptions about the signals under study. The only strong hypothesis is that the corrupting noise is stationary and stays stationary during the decomposition process by EMD. We have shown improved results with respect to the algorithm for signals having very different characteristics. We have demonstrated that our algorithm is robust with respect to noise for all of the studied signals. A de-mixing step is used in the algorithm. We have shown, specifically for the synthetic signals, that this step may not lead to an improvement, depending on the application, i.e. depending on whether the signal is simply to be reconstructed or if a further analysis of the individual IMFs using Hilbert spectrum is the objective of the processing. The evaluation of the proposed IMF likeness measure could indicate if this step is suitable for a further spectral analysis. If the measure obtained on the first denoised IMF is higher than the one obtained on the sum of this first denoised IMF and the second one, we would suggest not doing the step $3 \mathrm{~b}$. In this case, it is more suitable to add a new IMF corresponding to the first denoised one, 

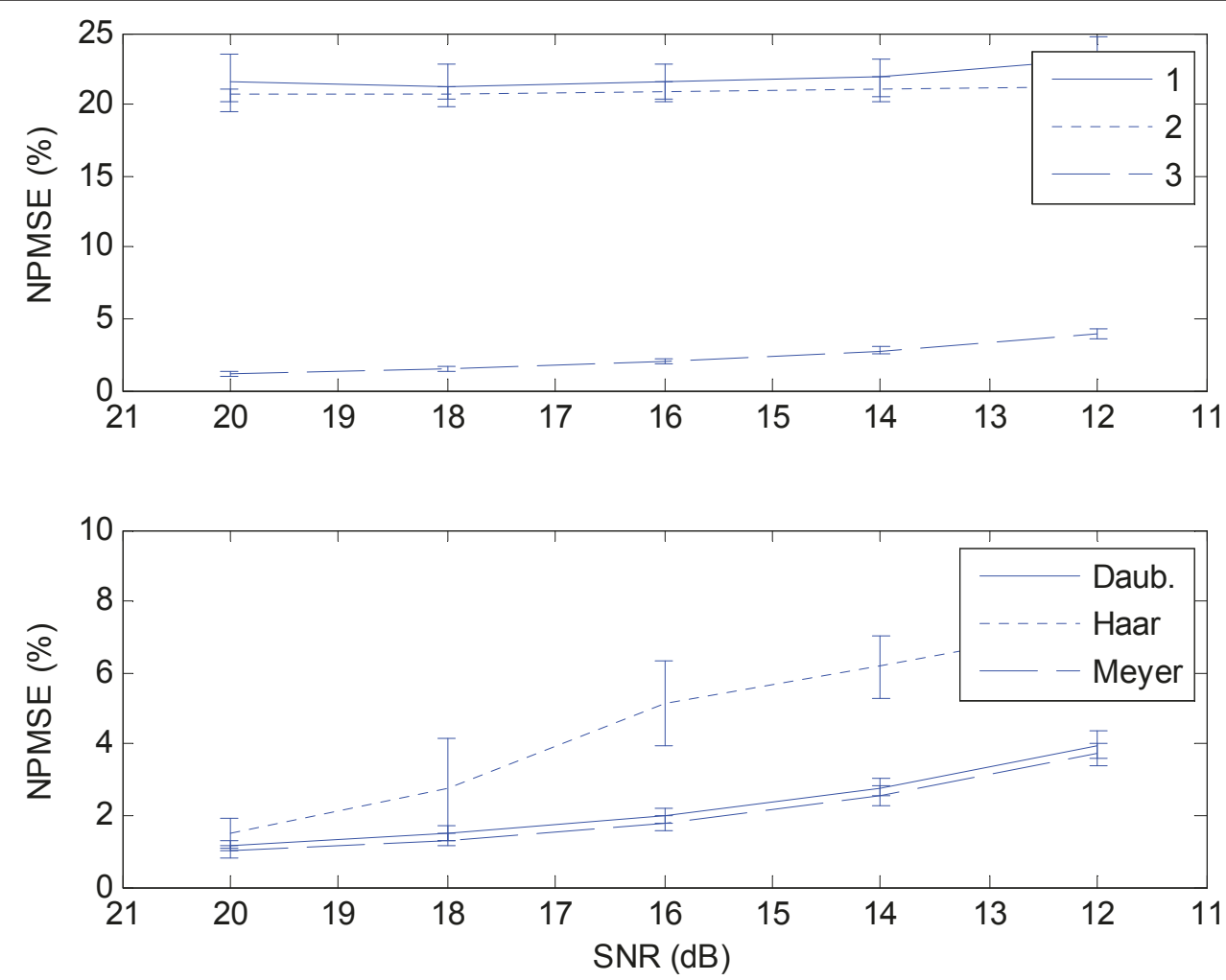

Figure 5 Results on the mechanical signal from the ball bearing plus noise. Above: The evolution of the percentage of mean square error (NPMSE) $( \pm \sigma)$ as obtained by using the classic method (1), by only correcting the estimate of noise in the first IMF (2) and by correcting the estimate of noise in the first IMF and plus modification of the second IMF (3). Below: Comparison of the NPMSE obtained using the wavelets Daubechie (Daub.), Haar and Meyer.
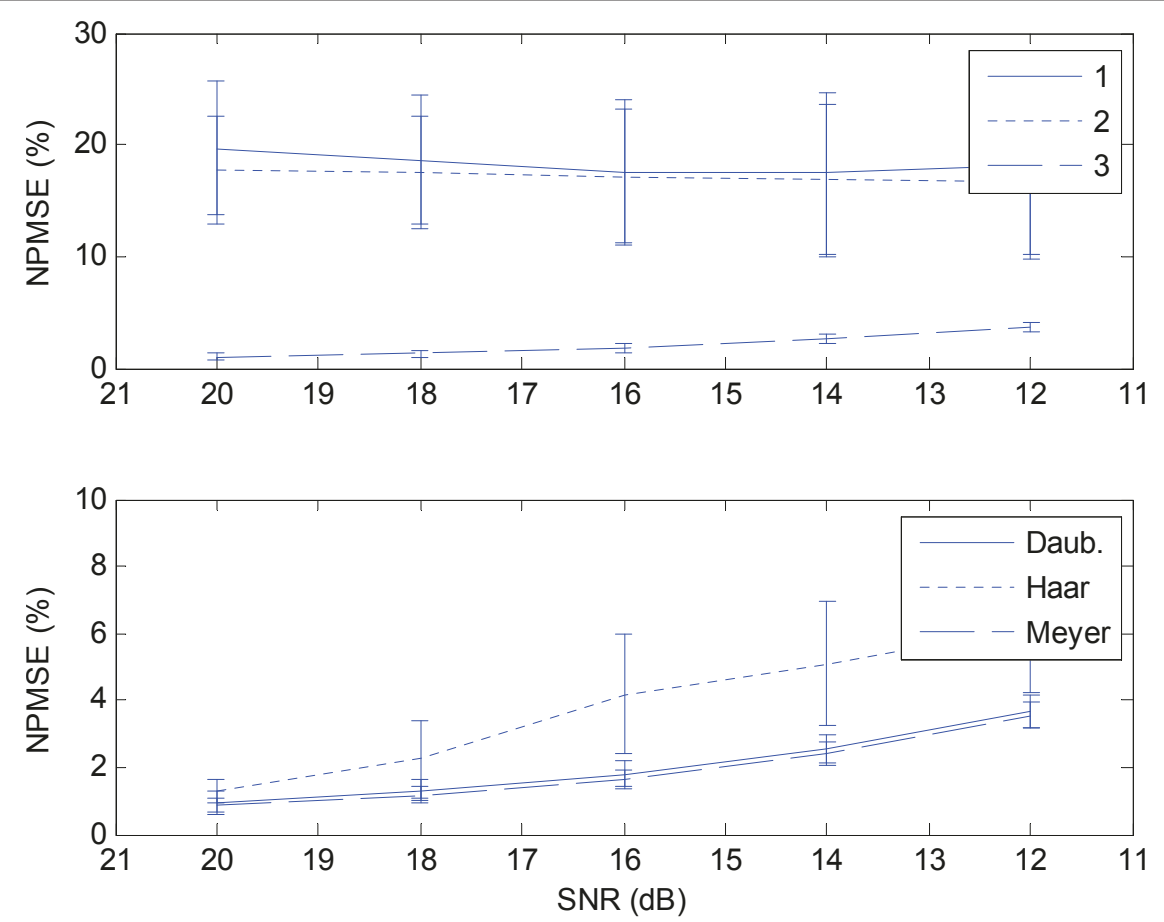

Figure 6 Results on the ECG signal plus noise. Above: The evolution of the percentage of mean square error (NPMSE) ( $\pm \sigma$ ) as obtained by using the classic method (1), by only correcting the estimate of noise in the first IMF (2) and by correcting the estimate of noise in the first IMF and plus modification of the second IMF (3). Below: Comparison of the NPMSE obtained using the wavelets Daubechie (Daub.), Haar and Meyer. 
Table 1 Median of the relative energy (lower quartileupper quartile), expressed as a percentage of the first IMF obtained after EMD of the: first denoised IMF (A), second IMF + first denoised IMF (B)

\begin{tabular}{lcc}
\hline Signal & A & B \\
\hline Synth. & $88.4 \%(81.9-93.3)$ & $81.7 \%(78.1-85.2)$ \\
Meca. & $74.3 \%(68.9-88.5)$ & $90.3 \%(79.6-92.1)$ \\
ECG & $42.5 \%(36-49)$ & $85.8 \%(74.4-91)$ \\
\hline
\end{tabular}

Results for the synthetic signal (Synth), mecanic signal (Meca), and the ECG signal.

before computing the Hilbert-Huang spectrum of the signal of interest. A deeper analysis of the reasons of these losses of IMF characteristics may help to define more efficient strategies in order to further improve the spectral estimation of the signals of interest.

List of abbreviations

EMD: empirical mode decomposition; IMF: intrinsic mode functions.

\section{Acknowledgements}

This work was supported by the Icelandic center for the research «RANNIS».

\section{Author details}

'Department of electronics, Université de Technologie de Compiègne, Compiègne Cedex, F60205, France ${ }^{2}$ UMR CNRS 6600, Université de Technologie de Compiègne, Compiègne Cedex, F60205, France ${ }^{3}$ School of Science and Engineering, Reykjavik University, Menntavegur 1, Reykjavik 103, Iceland

\section{Competing interests}

The authors declare that they have no competing interests.

Received: 10 December 2010 Accepted: 8 August 2011

Published: 8 August 2011

\section{References}

1. NE Huang, Z Shen, SR Long, MC Wu, HH Shih, O Zheng, N Yen, CC Tung, HH Liu, The empirical mode decomposition and the hilbert spectrum for nonlinear and non-stationary time series analysis. Proc R Soc Math Phys Eng Sci. 454, 903-995 (1998). doi:10.1098/rspa.1998.0193

2. Z Wu, NE Huang, A study of the characteristics of white noise using the empirical mode decomposition method. Proc R Soc Math Phys Eng Sci. 460, 1597-1611 (2004). doi:10.1098/rspa.2003.1221

3. P Flandrin, P Goncalves, G Rilling, in EMD Equivalent Filter Banks, from Interpretation to Applications, ed. by NE Huang, SSP Shen Hilbert-Huang Transform and Its Applications (World Scientific, Singapore, 2005), pp. 57-74

4. R Deering, JF Kaiser, The use of a masking signal to improve empirical mode decomposition. in IEEE International Conference on Acoustics, Speech, and Signal Proceedings, Philadelphia, PA, USA, (2005)

5. M Blanco-Velasco, B Weng, KE Barner, ECG signal denoising and baseline wander correction based on the empirical mode decomposition. Comput Biol Med. 38, 1-13 (2008). doi:10.1016/j.compbiomed.2007.06.003

6. J Xiao, P Borgnat, P Flandrin, Testing stationarity with time-frequency surrogates. in XVth European Signal Proceedings Conference, Poznan, Poland, (2007)

7. PE McSharry, GD Clifford, L Tarassenko, LA Smith, A dynamical model for generating synthetic electrocardiogram signals. IEEE Trans Biomed Eng. 50, 289-294 (2003). doi:10.1109/TBME.2003.808805

doi:10.1186/1687-6180-2011-37

Cite this article as: Terrien et al:: Automatic detection of mode mixing in empirical mode decomposition using non-stationarity detection: application to selecting IMFs of interest and denoising. EURASIP Journal on Advances in Signal Processing 2011 2011:37.

\section{Submit your manuscript to a SpringerOpen ${ }^{\mathcal{O}}$ journal and benefit from:}

- Convenient online submission

- Rigorous peer review

- Immediate publication on acceptance

- Open access: articles freely available online

- High visibility within the field

- Retaining the copyright to your article

Submit your next manuscript at $>$ springeropen.com 